\title{
Towards free-standing graphane: atomic hydrogen and deuterium bonding to nano- porous graphene
}

\author{
Mahmoud Mohamed Saad Abdelnabi ${ }^{1}\left(\mathbb{D}\right.$, Elena Blundo ${ }^{1}(\mathbb{B}$, \\ Maria Grazia Betti ${ }^{2}$ (1) , Gianluca Cavoto $^{2}$ (1) , Ernesto Placidi $^{1}$ (1), \\ Antonio Polimeni ${ }^{1}$ (1), Alessandro Ruocco ${ }^{3}$ (1), Kailong $\mathrm{Hu}^{4}$ (1), \\ Yoshikazu Ito ${ }^{4}$ (i) and Carlo Mariani ${ }^{2}$ (i)

\footnotetext{
${ }^{1}$ Dipartimento di Fisica, Sapienza Università di Roma, P.le Aldo Moro 2, 00185 Rome, Italy

${ }^{2}$ Dipartimento di Fisica and INFN Sezione di Roma 1, Sapienza Università di Roma, P.le Aldo Moro 2, 00185 Rome, Italy 00146 Rome, Italy

${ }^{4}$ Institute of Applied Physics, Graduate School of Pure and Applied Sciences, University of Tsukuba, Tsukuba 305-8573, Japan
} \\ ${ }^{3}$ Dipartimento di Scienze and INFN Sezione di Roma 3, Università di Roma Tre, Via della Vasca Navale, \\ E-mail: carlo.mariani@uniroma1.it
}

Received 19 June 2020, revised 14 September 2020

Accepted for publication 5 October 2020

Published 22 October 2020

\begin{abstract}
Graphane is formed by bonding hydrogen (and deuterium) atoms to carbon atoms in the graphene mesh, with modification from the pure planar $\mathrm{sp}^{2}$ bonding towards an $\mathrm{sp}^{3}$ configuration. Atomic hydrogen (H) and deuterium (D) bonding with $\mathrm{C}$ atoms in fully freestanding nano porous graphene (NPG) is achieved, by exploiting low-energy proton (or deuteron) non-destructive irradiation, with unprecedented minimal introduction of defects, as determined by Raman spectroscopy and by the $\mathrm{C} 1 \mathrm{~s}$ core level lineshape analysis. Evidence of the H- (or D-) NPG bond formation is obtained by bringing to light the emergence of a H- (or $\mathrm{D}-$ ) related $\mathrm{sp}^{3}$-distorted component in the $\mathrm{C} 1 \mathrm{~s}$ core level, clear fingerprint of $\mathrm{H}-\mathrm{C}$ (or D-C) covalent bonding. The $\mathrm{H}$ (or D) bonding with the $\mathrm{C}$ atoms of free-standing graphene reaches more than $1 / 4$ (or 1/3) at\% coverage. This non-destructive H-NPG (or D-NPG) chemisorption is very stable at high temperatures up to about $800 \mathrm{~K}$, as monitored by Raman and x-ray photoelectron spectroscopy, with complete healing and restoring of clean graphene above $920 \mathrm{~K}$. The excellent chemical and temperature stability of H- (and D-) NPG opens the way not only towards the formation of semiconducting graphane on large-scale samples, but also to stable graphene functionalisation enabling futuristic applications in advanced detectors for the $\beta$ spectrum analysis.
\end{abstract}

Supplementary material for this article is available online

Keywords: graphane, nano porous graphene, hydrogenation, deuteration, XPS, Raman

(Some figures may appear in colour only in the online journal)

\section{Introduction}

One of the main aims of graphene functionalisation is to achieve a two-dimensional (2D) material with the analogous mechanical properties and morphological aspect of graphene, while modifying its electronic response. In particular, graphane is the predicted $2 \mathrm{D}$ extended form of a covalently bonded hydrocarbon [1], which maintains the continuous 2D morphology of graphene, but where the pristine pure $\mathrm{sp}^{2}$-bonded $\mathrm{C}$ lattice mesh is modified by $\mathrm{H}-\mathrm{C} \mathrm{sp}^{3}$ bond 
distortion. The main consequence is a semiconducting character of graphane, whose actual energy gap $(\geqslant 3.5 \mathrm{eV})$ is predicted to depend on the hydrogen coverage and on the specific $\mathrm{H}-\mathrm{C}$ spatial configuration [1-3].

The semiconductor character of graphane, while maintaining the mechanical characteristics of graphene, constitutes one of its potential uses as part of different potential devices, ranging from catalysis to sensors to electronics, like other nano-structured materials [4-10], as also as a new material for the chemical storage of hydrogen [11]. However, recently the use of graphene functionalised with tritium (T) $-\mathrm{a} \beta$-unstable isotope of hydrogen-has been proposed as one of the basic and crucial elements of a new concept spectrometer able to study the endpoint of the tritium $\beta$-electron spectrum. This conceptual detection scheme (named Ptolemy) [12], in which some of us are involved, would have an unprecedented accuracy in the determination of the electron kinetic energy and would be able to sustain tritium activities well above the state of the art. It would eventually be able to largely improve the sensitivity of a measurement of the mass of the electron neutrino. A futuristic version of it-hosting up to $100 \mathrm{~g}$ of tritium-would even be able to detect the cosmological neutrino background, created at a very early age of our Universe, few seconds after the Big Bang [13]. A key aspect of the Ptolemy concept is the availability of a technique to obtain an efficient stabilisation of $\mathrm{T}$ by chemisorbing it on graphene. However, the handling of $\mathrm{T}$ in its gas phase presents critical safety issues and requires special radio-protected facilities. Thanks to the same chemical reactivity expected for atomic tritium with the lighter hydrogen and deuterium isotopes towards the $\mathrm{C}$ atoms, in the present work we chemisorb $\mathrm{H}$ and $\mathrm{D}$ on graphene to simulate in a safe way this chemical process. In this way we aim at testing both $\mathrm{H}$ and $\mathrm{D}$ chemical bonding to graphene as also the temperature stability of graphane, a crucial issue in view of the perspective extension to a tritium-based detector.

Atomic hydrogen covalent bonding to $\mathrm{C}$ atoms in the $\mathrm{sp}^{2}$ lattice mesh requires deformation towards an $\mathrm{sp}^{3}$ bond, with a pull-out of the $\mathrm{C}$ atom bonded to $\mathrm{H}$ by about $0.3 \AA$ from the average graphene plane. It is remarkable that the $\mathrm{H}$ chemisorption energy barrier decreases to $\sim 0.2 \mathrm{eV}$ [14] from 0.4-to$0.6 \mathrm{eV}$ [15-17], thanks to the local curvature of the hexagonal $\mathrm{C}$ lattice [18]. Thus, intrinsic graphene rippling may strongly favour atomic hydrogen chemisorption.

There has been a wide effort to experimentally achieve graphane in the last years, by adopting a variety of hydrogenation methods (from hot hydrogen plasma, to cold hydrogen plasma [19], to ion impinging, to high-temperature molecular hydrogen cracking) and on a number of graphene sample qualities/shapes, from graphite-exfoliated slivers [17, 19-21], to chemical-vapour-deposition (CVD) synthesised and transferred graphene flakes [22-24], to in situ grown metal-supported mono-layer graphene [25-29]. In most of these works, the $\mathrm{H}$ uptake on graphene has been inferred by Raman spectroscopy, detecting changes in the Raman band distribution and relative intensity, and sometimes by the $\mathrm{C} 1 \mathrm{~s}$ core level lineshape, with the emergence of the $\mathrm{sp}^{3}$-like component, clear signature of bond deformation.
However, the temperature stability of hydrogenated graphene is still an open issue, insofar de-hydrogenation has been observed to begin in a range between 350 [17] and $470 \mathrm{~K}$ [20], while complete dehydrogenation with restored pristine graphene properties has been obtained in a temperature range between $570-580 \mathrm{~K}[23,24]$ and $620-770 \mathrm{~K}$ $[11,17,19]$, these wide ranges strongly depending on both the hydrogenation method and the sample form/preparation. Thus, to obtain graphane starting from an actual self-standing high-quality graphene, with optimised exposure conditions to achieve a high H (D) upload, and with a good thermal stability is still an open question, with paramount importance in view of the perspective $\mathrm{T}$ stabilisation on graphene.

Nano-porous graphene (NPG) is a graphene sheet constituted by a very compact, bi-continuous interconnected three-dimensional (3D) arrangement of high-quality graphene [30, 31]. Raman and photoemission valence band and core level spectroscopy of NPG allow to consider it a very highquality one to weakly interacting turbostratic few-layers graphene [31], presenting different intrinsic curvatures at the scale of hundreds of $\mathrm{nm}$ whose spectroscopic fingerprints we have recently fully brought to light $[31,32]$. Thus, NPG represents a realistic system of free-standing unsupported graphene, where it should be possible to achieve hydrogenation (or deuteration) towards 3D compact and bulkscalable graphane in very clean vacuum conditions and where one can assess the chemical bonding and the thermal stability of hydrogenated (or deuterated) free-standing graphene.

In this paper, we achieve hydrogenated (and deuterated) NPG by in-vacuum low-energy proton (or deuteron) irradiation, with a saturation around $1 / 4$ at $\% \mathrm{C}(1 / 3$ at $\% \mathrm{C})$ of full coverage, with the presence of $\mathrm{H}-\mathrm{C}$ (and $\mathrm{D}-\mathrm{C}$ ) bonding and with a very high thermal stability of the hybridisation, as determined by Raman and x-ray photoelectron spectroscopy (XPS). The high thermal stability of both H-NPG and D-NPG can open the way to the potential development of NPG not only as a stable semiconductor, but also as a compact highdensity host for perspective atomic $\mathrm{T}$ stabilisation and future advanced detectors [12].

\section{Experimental methods}

The NPG samples were synthesised by a nano-porous Nibased CVD method [30, 33-35]. $\mathrm{Ni}_{30} \mathrm{Mn}_{70}$ ingots were prepared by melting pure $\mathrm{Ni}$ and $\mathrm{Mn}$ (purity $>99.9$ at\%) using an Ar-protected arc melting furnace. After annealing at $900{ }^{\circ} \mathrm{C}$ for $24 \mathrm{~h}$ for microstructure and composition homogeneisation, the ingots were cold-rolled to thin sheets $(50 \mu \mathrm{m})$ at room temperature (RT). Nano-porous $\mathrm{Ni}$ was prepared from the $\mathrm{Ni}_{30} \mathrm{Mn}_{70}$ sheet by chemical dealloying in a $1.0 \mathrm{M}\left(\mathrm{NH}_{4}\right)_{2} \mathrm{SO}_{4}$ aqueous solution at $50{ }^{\circ} \mathrm{C}$ for overnight. After the dealloying, the samples were rinsed thoroughly with distilled water and then rinsed with ethanol before dried in vacuum. The resulting nano-porous $\mathrm{Ni}$ substrates loaded in a quartz tube $(\phi 26 \times \phi 22 \times 250 \mathrm{~mm})$ were inserted into a quartz tube $(\phi 30 \times \phi 27 \times 1000 \mathrm{~mm})$ furnace and annealed at $800{ }^{\circ} \mathrm{C}$ under flowing gas mixed by $200 \mathrm{sccm} \mathrm{Ar}$ and $100 \mathrm{sccm} \mathrm{H}_{2}$ 
for $3 \mathrm{~min}$. After the reduction pre-treatment, a mixed atmosphere of $\mathrm{H}_{2} \quad(100 \mathrm{sccm})$, Ar $(200 \mathrm{sccm})$ and benzene (0.5 mbar, 99.8\%, anhydrous, Ardrich) was introduced for graphene growth at $800{ }^{\circ} \mathrm{C}$ for $90 \mathrm{~s}$. Subsequently, the furnace was opened for quick cooling of the inner quartz tube. The nanoporous $\mathrm{Ni}$ substrates were dissolved by $1.0 \mathrm{M} \mathrm{HCl}$ solution and then transferred into $2.0 \mathrm{M} \mathrm{HCl}$ solution to completely removed residual $\mathrm{Ni}$. The samples were repeatedly washed with water and kept in water.

Hydrogenation (deuteration) of the NPG samples was achieved by using a Kaufman source to irradiate the samples with a low energy proton (deuteron) beam [36, 37]. To do that, the sample was first mounted into a vacuum chamber by means of a metallic holder, in such a way to guarantee the thermal and electrical (ground) contact. Different experimental conditions during irradiation were tested, like beam energy up to $20 \mathrm{eV}$, different pressures and doses, and also irradiation with the sample kept at high temperature. The choice of such low beam energies is grounded on previous studies on other layered materials (transition-metal dichalcogenides and post-transition-metal chalcogenides), showing how the irradiation process did not result in the formation of defects, but rather in the incorporation of hydrogen in either molecular or atomic form [37-39]. We tested several NPG samples with different conditions (ion energy, dose, pressure, sample temperature), and the best conditions for the highest $\mathrm{H}$ (D) upload in graphene were obtained with the chamber pressure set at about $1 \times 10^{-6}$ mbar, the sample kept at RT, and protons (deuterons) produced in an ionisation chamber and accelerated by a system of grids in such a way to irradiate the sample with ion current density of $0.5 \mu \mathrm{A} \mathrm{cm}^{-2}$ and energy beams of $6 \mathrm{eV}$.

A same large flake (figure 1(a)) was divided into two parts, to have similar and homogeneous samples to be hydrogenated and deuterated. The total $H$ (D) dose can be quantified as the areal density (number of ions $\mathrm{cm}^{-2}$ ) of $\mathrm{H}^{+}$ $\left(\mathrm{D}^{+}\right)$ions impinging on the sample surface. Irradiation was performed until saturation of $\mathrm{H}$ (D) bonding has been detected by XPS (see supplementary data, SD, figure S1 available online at stacks.iop.org/NANO/32/035707/mmedia), with a final exposure of $(5.5 \pm 0.3) \times 10^{16}$ ions $\mathrm{cm}^{-2}$ and $(5.8 \pm 0.8) \times 10^{16}$ ions $\mathrm{cm}^{-2}$ for $\mathrm{H}$ and $\mathrm{D}$, respectively. After each exposure, the sample was dry transferred and directly measured by XPS in ultra-high-vacuum (UHV).

The XPS experiments were carried out at the Nanostructures at Surfaces laboratory at Sapienza University in Rome, in an UHV chamber with base pressure in the low $10^{-10}$ mbar range. An $\mathrm{x}$-ray photon source with $h \nu=1253.6 \mathrm{eV}$ was used (PSP TA10 $\mathrm{Mg} \mathrm{K}_{\alpha}$ ), photoelectrons were measured with a hemispherical VG Microtech Clam-2 electron analyser in constant pass energy mode set at $50 \mathrm{eV}$ and with overall energy resolution of $\leqslant 1 \mathrm{eV}$; further experimental details are available in [40-43]. The binding energy (BE) scale was calibrated on a freshly sputtered gold foil in electrical contact with the sample, by setting the $\mathrm{Au}$ $4 \mathrm{f}_{7 / 2}$ core-level at $84.0 \mathrm{eV} \mathrm{BE}$.

The Raman experiments were carried out at the Optical Spectroscopy of Nanostructured Materials laboratory
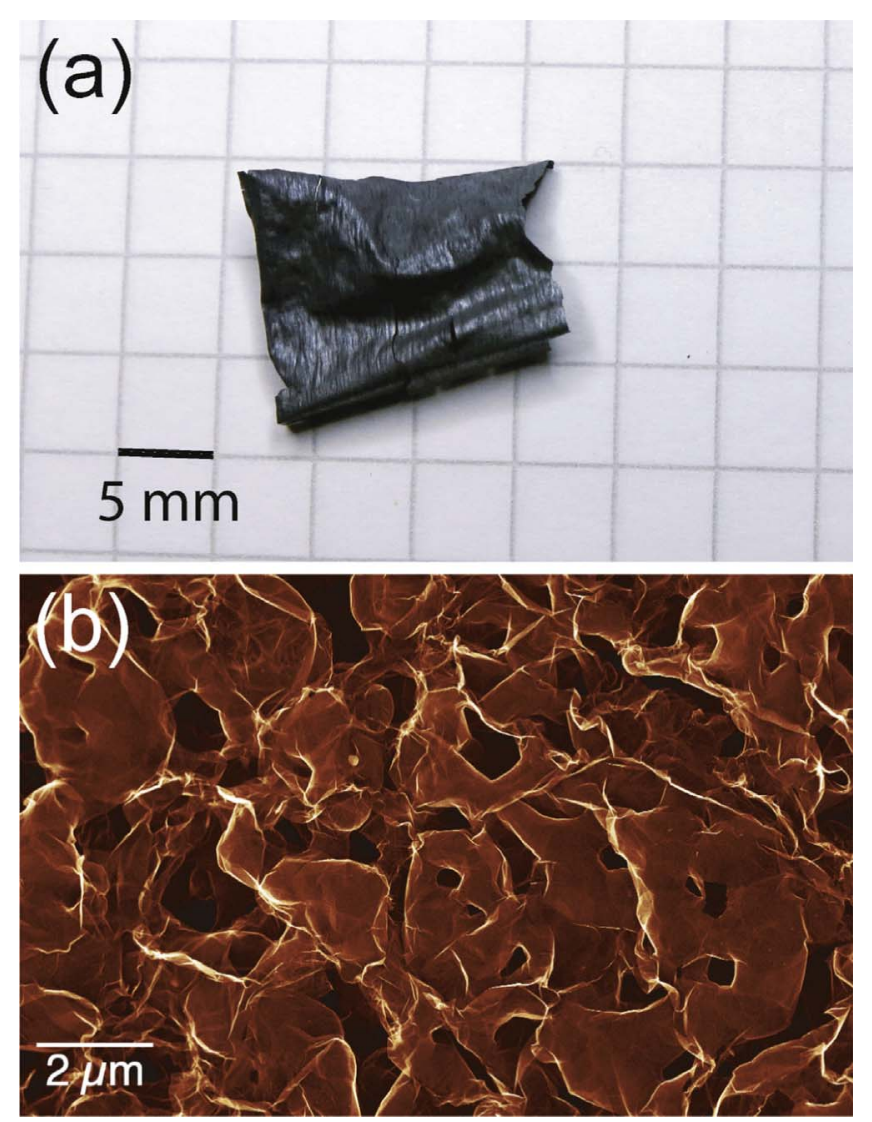

Figure 1. (a) Picture of $\mathrm{a} \mathrm{cm}^{2}$-sized NPG sample; (b) SEM image of the NPG sample.

(Sapienza University, Rome). The excitation laser was provided by a single frequency Nd:YVO4 lasers (DPSS series by Lasos) with emission wavelength equal to $532.2 \mathrm{~nm}$. A confocal microscope equipped with a Zeiss $100 \times$ objective $(\mathrm{NA}=0.75)$ or with a Leitz $10 \times$ objective $(\mathrm{NA}=0.2)$ was used to excite and collect the light, in a backscattering configuration [44]. In order to avoid sample damage, a moderate laser power was used, $\leqslant 50 \mu \mathrm{W}$ measured at the entrance of the objective. We checked that such a laser power was not inducing changes in the spectra. The signal was spectrally analysed with by a $750 \mathrm{~mm}$ focal length monochromator (ACTON SP750) equipped with a 1200 groove $\mathrm{mm}^{-1}$ grating and detected by a back-illuminated Si CCD Camera (model 100BRX by Princeton Instruments), and the reflected laser light was filtered out by a very sharp high-pass Razor edge filter at $535 \mathrm{~nm}$ (Semrock). The spectral resolution of our Raman setup was $0.7 \mathrm{~cm}^{-1}$. The Raman band intensities and experimental uncertainties have been derived by a Lorentzian fitting of the data.

The scanning electron microscopy (SEM) images were taken at the CNIS laboratory of Sapienza University, Rome, with a field-emission Zeiss Auriga 405 instrument, with a nominal resolution of $1 \mathrm{~nm}$ at maximum magnification, with a beam energy of $10 \mathrm{keV}$, and at a working distance of about $3.5 \mathrm{~mm}$. 

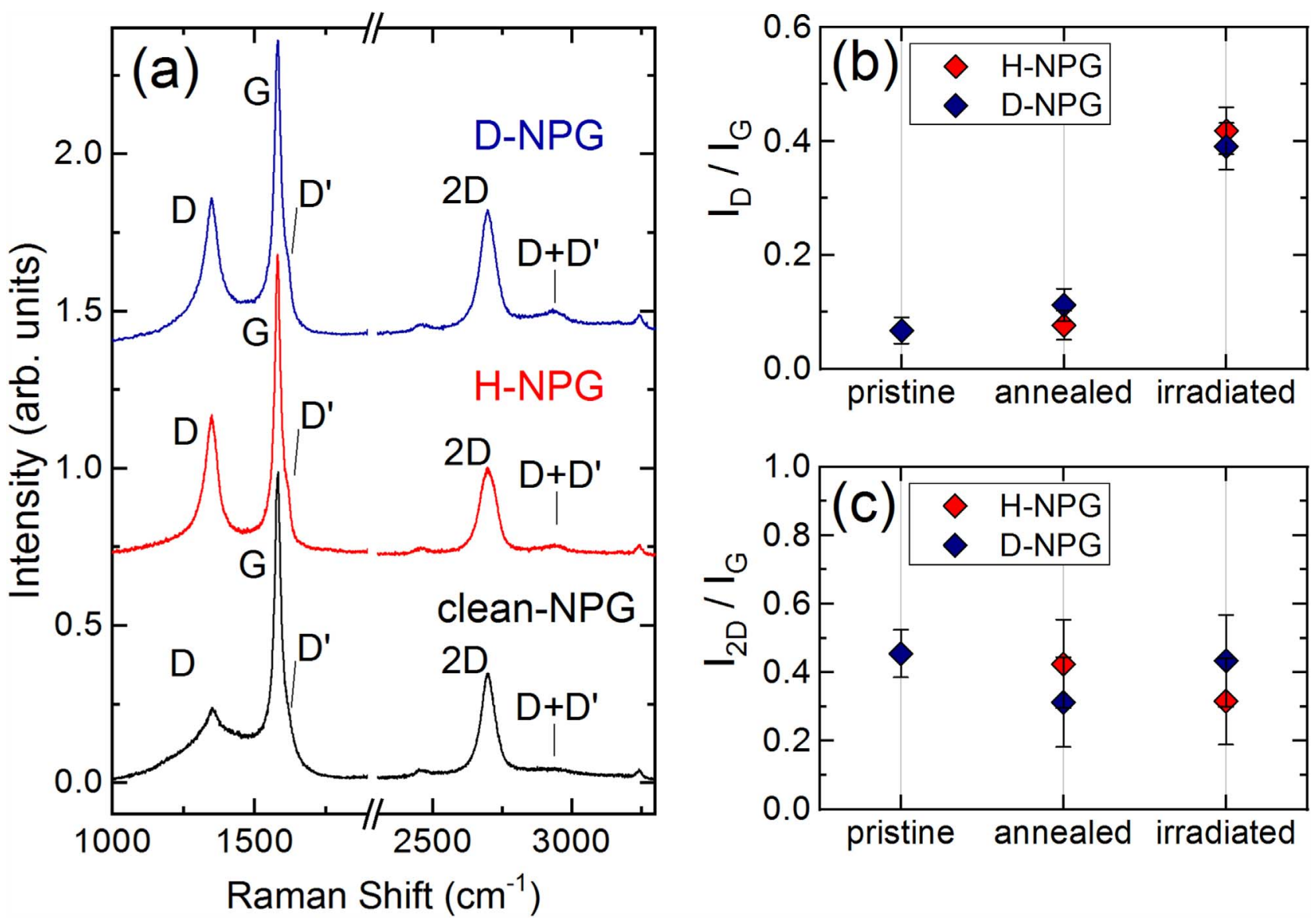

Figure 2. (a) Raman spectra taken on clean annealed NPG (bottom spectrum), H-saturated NPG (middle spectrum) and D-saturated NPG (top spectrum); data normalised to the $G$ peak height and vertically stacked for the sake of clarity; the spectra were acquired by focusing a $532.2 \mathrm{~nm}$ laser by means of a $100 \times$ objective with $\mathrm{NA}=0.75$, resulting in a laser power density $\leqslant 50 \mu \mathrm{W}$ at the entrance of the objective. Intensity ratio between the $I_{\mathrm{D}} / I_{\mathrm{G}}(\mathrm{b})$ and $I_{2 \mathrm{D}} / I_{\mathrm{G}}$ (c) Raman bands in the pristine, annealed and $\mathrm{H}(\mathrm{and} \mathrm{D})$ irradiated samples; the experimental uncertainty on the intensity ratios is derived from the Lorentzian fitting results.

\section{Results and discussion}

The NPG samples used for the hydrogenation/deuteration experiments are wide graphene samples, around $(1.2 \times 1.2) \mathrm{cm}^{2}$ sized and $30 \mu \mathrm{m}$ in thickness (figure $\left.1(\mathrm{a})\right)$, with a light woolly aspect, being composed by 3D compact and bi-continuous graphene, with an average pore-size of few hundreds of nm [30], as visible in the SEM image shown in figure 1(b). The same NPG samples were divided into two parts, to obtain similar and homogeneous samples from the same batch to be hydrogenated or deuterated. Each NPG sample has been pre-treated by $920 \mathrm{~K}$ annealing in UHV for desorbing adventitious contaminants, an important step to achieve a clean NPG to be efficiently protonated and deuterated (see SD, figure S2).

The Raman spectrum of the clean NPG sample after annealing (figure 2(a), lower spectrum) presents the expected 2D and $G$ bands of high-quality graphene at 2896 and $1581 \mathrm{~cm}^{-1}$, respectively, with a very small defect peak D at $1350 \mathrm{~cm}^{-1}$, pointing to a very low density of lattice defects, whose presence can be rather associated to the small defect density at bent regions and acute curvatures of the nanoporous structure [31]. There is a low structureless bump below the main bands, due to a slight amorphous signal due to the annealing process necessary to clean the sample (see SD, figure S2). The relative Raman band intensity of this graphene continuous structure addresses the presence of bi-layer and few-layers graphene, and the lineshape indicates the presence of a turbostratic stacking, suggesting a weak bonding between the planes [31, 32]. An accurate analysis of the topology, structural, vibrational, electronic insights of pristine NPG is discussed in detail in our previous works [30-33].

The C 1s core-level measured on the NPG sample is shown in figure 3 (lower spectrum), along with the results of a fitting analysis. Data have been fitted with Voigt lineshaped components, i.e. Lorentzian-Gaussian curves which take into account the intrinsic linewidth and experimental uncertainty, respectively, with the exception of the main $\mathrm{sp}^{2}$ component which possesses an asymmetric Doniach-Sunjic lineshape, with asymmetry parameter $\leqslant 0.1[45-47]$ associated to the semimetallic nature of NPG. The C 1s lineshape is characterised by the main feature associated to the $\mathrm{sp}^{2}$ bonding at $284.6 \mathrm{eV} \mathrm{BE}$, with a slightly skewed tail at higher BE due to a small $\mathrm{sp}^{3}$ peak at $285.2 \mathrm{eV}$, associated to the slight bond deformation at wrinkled/bent regions of NPG as recently observed by spectro-microscopy experiments [31, 32]; the 


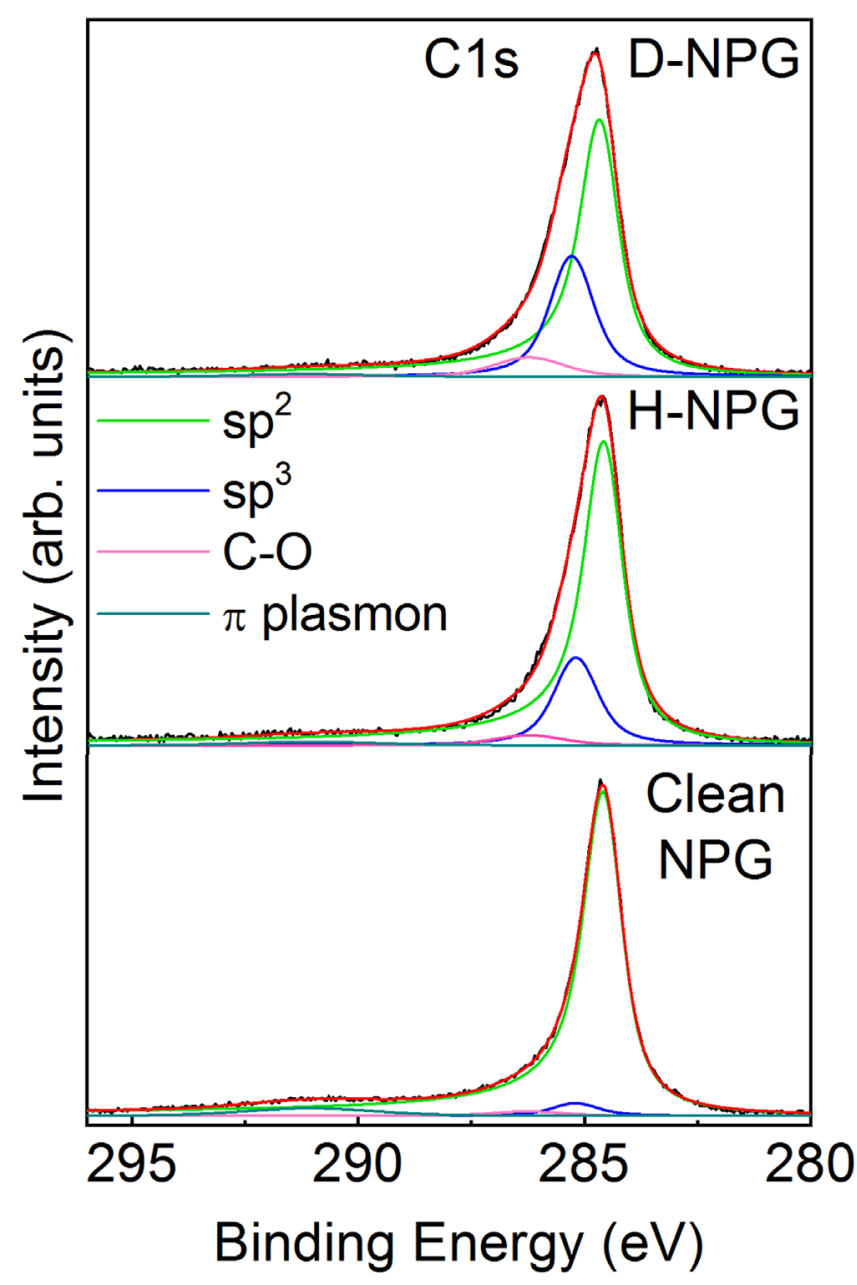

Figure 3. C 1s XPS spectra for clean NPG after annealing (bottom spectrum) and for NPG exposed to saturation coverage of $\mathrm{H}$ (middle spectrum) and D (top spectrum); experimental data (black lines), $\mathrm{sp}^{2}$ fitting component (green lines), $\mathrm{sp}^{3}$ component (blue lines), C-to-O component (pink lines), $\pi$-plasmon excitation (light-blue lines) and fitting sum curve (red lines); $\mathrm{H}^{+}$exposure of $5.5 \times 10^{16}$

protons $\mathrm{cm}^{-2}$ at $6 \mathrm{eV} \mathrm{H}^{+}$energy, $\mathrm{D}^{+}$exposure of $5.8 \times 10^{16}$ deuterons $\mathrm{cm}^{-2}$ at $6 \mathrm{eV} \mathrm{D}^{+}$energy.

broad component at about $291.1 \mathrm{eV}(6.5 \mathrm{eV}$ from the main peak) is due to the plasmon associated to the $\pi$ collective excitation [48-50], while the component centred at about $286.5 \mathrm{eV}$ is ascribed to a small residual C-to-O structure [51-53].

The NPG sample has been exposed to either H or D ions till saturation, and the corresponding Raman spectra of H-saturated NPG (H-NPG) and D-saturated NPG (D-NPG) are displayed in figure 2(a), along with the $\mathrm{D} / \mathrm{G}$ (figure 2(b)) and $2 \mathrm{D} / \mathrm{G}$ (figure 2(c)) intensity ratios obtained by fitting the Raman bands with Lorentian curves. The 2D/G Raman band intensity ratios do not change much (within 10\%) with respect to the clean annealed NPG, thus indicating that $\mathrm{H}$ and D functionalisation of $\mathrm{C}$ atoms does not alter the few-layers graphene sheet quality. On the other hand, the Raman bands associated to defects $\left(\mathrm{D}, \mathrm{D}^{\prime}\right.$ and $\left.\mathrm{D}+\mathrm{D}^{\prime}\right)$ become more intense after hydrogenation (and deuteration) of NPG. The Raman band evolution is qualitatively in line with what previously observed on few-layers and substrate-supported graphene functionalisation via cold plasma [19] and other hydrogenation methods [17, 21, 25, 54-56], and attributed to $\mathrm{H}-\mathrm{C}$ bond distortion.

However, we remark that our low-energy ion hydrogenation (and deuteration) method of free-standing NPG induces a much lower defect peak $(\mathrm{D} / \mathrm{G}$ band intensity ratio $<1)$ than previously obtained with other methods and/or graphene sample qualities, for which a Raman D/G band intensity ratio $\gg 1$ (from 2.5 to 5) was measured $[17,19,22,25,54]$. Thus, the present in-vacuum and lowenergy [57] $\mathrm{H}^{+}$and $\mathrm{D}^{+}$ion loading of NPG is an excellent viable, clean and low-defect non-destructive method towards atomic hydrogen and deuterium uploading in graphene.

A direct evidence of the $\mathrm{sp}^{3}$ bond establishment between $\mathrm{C}$ atoms and $\mathrm{H}$ (or D), though, can be obtained by analysing the $\mathrm{C} 1 \mathrm{~s}$ core level lineshape. The $\mathrm{C} 1 \mathrm{~s}$ XPS core level data taken at both H-saturated and D-saturated NPG are shown in figure 3 (medium and upper spectra), along with the results of a fitting analysis carried out by using the Voigt lineshaped curves previously discussed, whose values are reported in table 1 .

After hydrogenation and deuteration of NPG at saturation, all the components, namely the $\mathrm{sp}^{2}$ and $\mathrm{sp}^{3}$ peaks, the $\pi$-plasmon excitation and the small C-to-O residual peak, do not shift in energy within the experimental uncertainty derived by the fitting procedure, while their relative intensity is clearly modified. The $\mathrm{sp}^{3}$ peak increase leads to a $\Theta$ ratio of about $25 \pm 3 \%$ for $\mathrm{H} \quad(36 \pm 3 \%$ for $\mathrm{D})$, where $\Theta=I\left(\mathrm{sp}^{3}\right) /\left[I\left(\mathrm{sp}^{2}\right)+I\left(\mathrm{sp}^{3}\right)\right]$. This maximum intensity ratio reached after exposure constitutes the saturation value for this fully free-standing self-suspended NPG sample in clean and controlled condition, and is very stable against air-exposure as also as a function of temperature at least up to $800 \mathrm{~K}$. We remark the absence of any defect-related C 1s core-level component at $\mathrm{BE}$ lower than the $\mathrm{sp}^{2}$ peak, demonstrating the absence of unsaturated $\mathrm{C}$ dangling bonds at vacancy sites [7, 28, 58-61] and revealing the non-destructive hydrogenation (and deuteration) process.

The obtained atomic $\mathrm{H}$ and $\mathrm{D}$ to $\mathrm{C}$ high uploading percentage of full protonated (and deuterated) graphene is in agreement with the best results achieved so far for hydrogenated graphene in a variety of graphene morphologies, whether in form of transferred mono-layer flakes [17, 24] or substrate-supported [26, 28]. In particular, it is in excellent agreement with the saturation value estimated for $\mathrm{H}(25 \%)$ and $\mathrm{D}(35 \%)$ chemisorption on Au-intercalated graphene/Ni, where the different saturation value has been ascribed to a different kinetics and higher desorption barrier for $\mathrm{D}$ than for $\mathrm{H}$ due to quantum-mechanical zero-point energy effects related to the D-C and $\mathrm{C}-\mathrm{H}$ vibrations [62]. Furthermore, it constitutes a lower limit of the maximum percentage, being the XPS technique surface sensitive, thus measuring the component distribution at the NPG veil surface.

The $\pi$-plasmon excitation is an excellent fingerprint of the quality of graphene [48, 49], since it is associated to a collective excitation spread over the $\pi$-coordinated lattice. A zoomed plot of the $\pi$-plasmon excitation energy region together with the fitting result and its relative intensity 
Table 1. Fit results for the $\mathrm{C} 1 \mathrm{~s}$ core level for the NPG sample and after exposure to saturation coverage of $\mathrm{H}^{+}$and $\mathrm{D}^{+}$. Name of the component, binding energy ( $\pm 0.1 \mathrm{eV}, \pm 0.3 \mathrm{eV}$ for the $\pi$-plasmon), asymmetry parameter, full-width at half-maximum (FWHM, $\pm 0.1 \mathrm{eV}$ ) of the Gaussian component, relative intensity (peak areas, $\pm 5 \%$ ). Reported uncertainties are derived by the fitting procedure.

\begin{tabular}{|c|c|c|c|c|}
\hline \multicolumn{5}{|c|}{ Clean NPG } \\
\hline C 1s component & $\mathrm{BE}(\mathrm{eV})$ & Asymm. param. & FWHM (eV) & Area/Area ${ }_{\text {tot }}$ \\
\hline $\mathrm{sp}^{2}$ & 284.6 & 0.09 & 1.1 & 88.0 \\
\hline $\mathrm{sp}^{3}$ & 285.2 & 0 & 1.2 & 4.3 \\
\hline$\pi$-plasmon & 291.1 & 0 & 3.4 & 5.5 \\
\hline C-to-O & 286.5 & 0 & 2.2 & 2.2 \\
\hline \multicolumn{5}{|c|}{ H-saturated NPG } \\
\hline C 1 s component & $\mathrm{BE}(\mathrm{eV})$ & Asymm. param. & FWHM (eV) & Area/Area ${ }_{\text {tot }}$ \\
\hline $\mathrm{sp}^{2}$ & 284.6 & 0.09 & 1.1 & 71.3 \\
\hline $\mathrm{sp}^{3}$ & 285.2 & 0 & 1.2 & 24.0 \\
\hline$\pi$-plasmon & 291.1 & 0 & 3.4 & 2.0 \\
\hline C-to-O & 286.5 & 0 & 2.2 & 2.6 \\
\hline \multicolumn{5}{|c|}{ D-saturated NPG } \\
\hline C 1s component & $\mathrm{BE}(\mathrm{eV})$ & Asymm. param. & FWHM (eV) & Area/Area ${ }_{\text {tot }}$ \\
\hline $\mathrm{sp}^{2}$ & 284.6 & 0.09 & 1.1 & 59.1 \\
\hline $\mathrm{sp}^{3}$ & 285.3 & 0 & 1.2 & 33.7 \\
\hline$\pi$-plasmon & 291.2 & 0 & 3.4 & 1.5 \\
\hline C-to-O & 286.5 & 0 & 2.2 & 5.7 \\
\hline
\end{tabular}

evolution are displayed in figure 4 , right and lower panels, respectively. The $\pi$-plasmon peak intensity definitely decreases at $\mathrm{H}$ and $\mathrm{D}$ saturation coverage, thus reflecting the reduction of continuity in the $\pi$ bonding of graphene [50], further proof of increasing bond deformation towards $\mathrm{sp}^{3}$. The intensity reduction of the plasmon peak is $25 \%$ higher in the case of D-C chemisorption than for the $\mathrm{H}-\mathrm{C}$ case, thus confirming the higher coverage of deuterons than of protons. Further evidence of $\mathrm{sp}^{3}$ increase after hydrogenation can also be inferred by the high-energy $\pi+\sigma$ plasmon mode lineshape change (see SD, figure S3).

Finally, we tested the temperature stability of the $\mathrm{H}$ - and D-NPG chemical bonding, by in-vacuum annealing the saturated hydrogenated (and deuterated) samples. Annealing up to $800 \mathrm{~K}$ causes only a slight increase of the $\pi$-plasmon intensity (less for D than for $\mathrm{H}$ ), still much lower than in clean graphene, accompanied by a $\Theta$ ratio reduction by less than $10 \%$, while only after annealing above $920 \mathrm{~K}$ we observe a definite $\Theta$ ratio decrease, with full recovering of the $\pi$-plasmon and of the pristine NPG C 1s lineshapes, indicating complete desorption and healing of graphene.

Previous experiments of graphene uploaded with hydrogen by different methods, ranging from the seminal work with cold plasma deposition [19], to plasma generation $[17,24]$, to electron-beam-induced de-hydrogenation of molecular species [20], showed that annealing temperatures from about $380 \mathrm{~K}$ upwards are sufficient to start a clear process of de-hydrogenation, until recovering of pristine graphene is achieved above $680 \mathrm{~K}$ [17, 20, 23], $720 \mathrm{~K}$ [19] or $770 \mathrm{~K}$ at most [11]. Analogous partial recovering of pristine graphene was previously observed by high-vacuum annealing at least at $750 \mathrm{~K}$, for hydrogenated transferred graphene flakes [55], while much less stable $\mathrm{H}-\mathrm{C}$ bonding against temperature has been achieved for supported/transferred graphene hydrogenated with different methods (graphene healing temperature range between 570 and $580 \mathrm{~K}$ [23, 24] and 620-720 K [17, 19]).

Our experimental evidences bring to light the definitely much higher thermal stability of the $\mathrm{C}-\mathrm{H}$ and $\mathrm{C}-\mathrm{D}$ chemical bonds for the present vacuum in situ hydrogen (and deuterium) molecular dissociation and consequent incorporation in fully free-standing NPG.

\section{Conclusions}

Atomic hydrogenation and deuteration of NPG towards graphane formation have been achieved with a $\sim 25$ at $\%(\sim 36$ at\%) saturation value of $\mathrm{H}$ (or D) covalent bonding with $\mathrm{C}$, as estimated by the $\mathrm{C} 1 \mathrm{~s}$ core level lineshape analysis, bringing to light and quantifying the percentage of the $\mathrm{sp}^{3}$ bond of $\mathrm{C}$ with protons (or deuterons) with respect to the planar $\mathrm{sp}^{2}$ bonds. Although analogous high $\mathrm{H}$ (or D) up-take had been previously achieved on graphene flakes/sheets with different morphologies and with other exposure techniques $[17,22,25,54]$, we obtain a remarkable much lower presence of defects after irradiation. Thus, the in-vacuum cracking of molecular hydrogen (or deuterium) with low-energy ion irradiation of free-standing graphene is non-destructive and constitutes a very clean and stable method for chemically uploading protons (or deuterons) on graphene, as determined by XPS and Raman spectroscopy. Furthermore, the H-NPG 


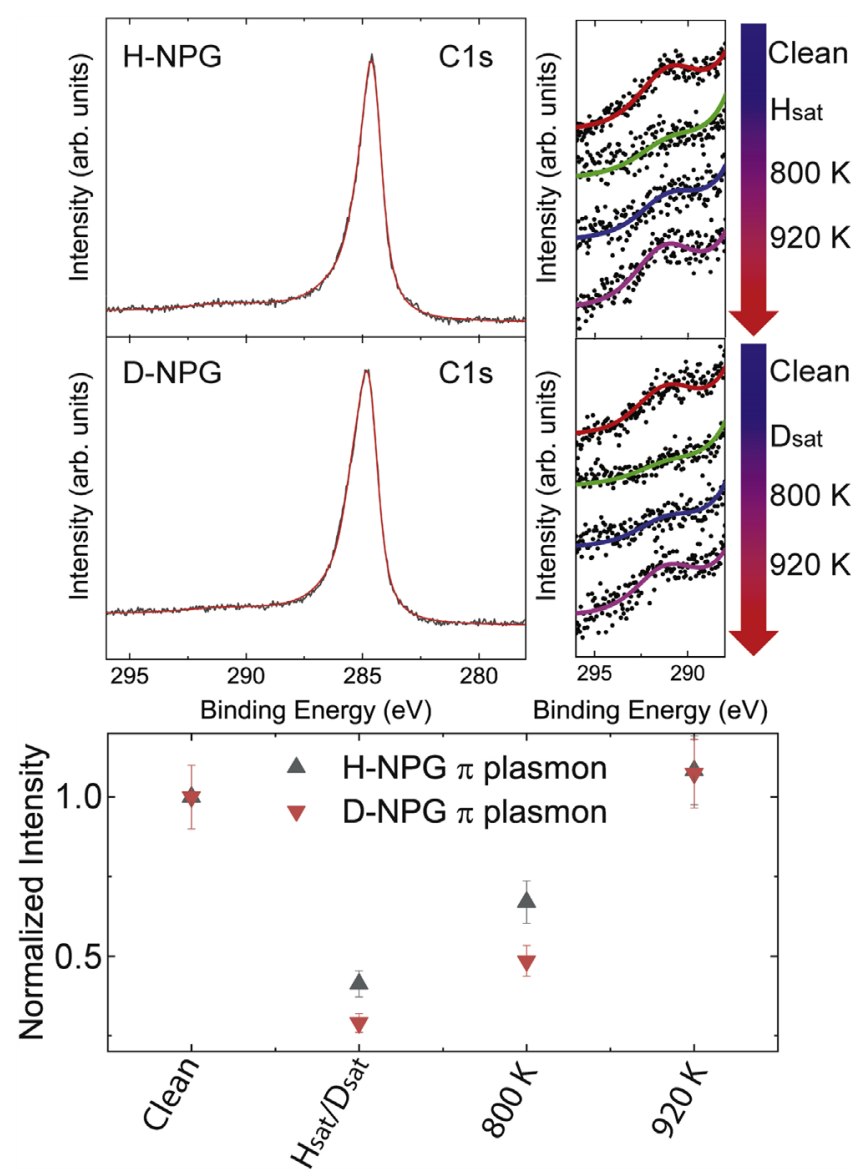

Figure 4. C 1s XPS spectra for H-NPG and D-NPG after annealing at $800 \mathrm{~K}$ (left panels); zoom of the $\pi$-plasmon excitation energy region for the clean, fully exposed $\left(\mathrm{H}_{\mathrm{sat}}\right.$ and $\left.\mathrm{D}_{\mathrm{sat}}\right)$ and annealed $(800 \mathrm{~K}$, $920 \mathrm{~K}$ ) samples (right panels); normalised intensity evolution of the $\pi$-plasmon excitation, as a function of exposure and temperature (bottom panel); experimental uncertainty derived by the fitting procedure.

and D-NPG percentages achieved at saturation coverage result to be very firm in temperature, with a $\mathrm{H}-\mathrm{C}$ - (or D-C-) related $\mathrm{sp}^{3}$ bonding component in the $\mathrm{C} 1$ s core level stable within $10 \%$ up to $\sim 800 \mathrm{~K}$, while healing of graphene is only achieved at unprecedented high temperatures ( $>900 \mathrm{~K})$, as observed by both the Raman and photoemission spectroscopic signatures and also demonstrated by the regained intensity of the $\pi$-plasmon excitation only above that high temperature.

Three main achievements have been reached in the present work: free-standing unsupported graphene sample of high quality can (i) upload a high percentage of hydrogen and deuterium (ii) with the best quality (lowest defect-density ever reached) and (iii) the highest temperature stability of graphane obtained so far. These evidences promote free-standing NPG and the in-vacuum protonation (and deuteration) via lowenergy ion deposition as an efficient method to stabilise the bonding of H-NPG (and D-NPG), thus paving the way for perspective future advanced applications, among which the use of the heavier isotope T-NPG as a potential element of a neutrino detector [12].

\section{Acknowledgments}

We thank Claudia Dentato and Francesco Mura for their experimental assistance in the XPS and SEM measurements, respectively. Work supported by PRIN Grant FERMAT (2017KFY7XF) from Italian Ministery MIUR, from Sapienza University Ateneo funds, from INFN Ptolemy project and from JSPS Grant-in-Aid for Scientific Research on Innovative Areas 'Discrete Geometric Analysis for Materials Design': Grant Number JP18H04477, JP20H04628.

\section{ORCID iDs}

Mahmoud Mohamed Saad Abdelnabi (i) https://orcid.org/ 0000-0002-0021-6181

Elena Blundo (iD https://orcid.org/0000-0003-0423-4798

Maria Grazia Betti (iD https://orcid.org/0000-0002-

6244-0306

Gianluca Cavoto (iD https://orcid.org/0000-0003-2161-918X

Ernesto Placidi (1D https://orcid.org/0000-0002-3820-8451

Antonio Polimeni (iD https://orcid.org/0000-0002-2017-4265

Alessandro Ruocco (i) https://orcid.org/0000-0002-

4909-7216

Kailong Hu (1) https://orcid.org/0000-0003-0489-5836

Yoshikazu Ito (iD) https://orcid.org/0000-0001-8059-8396

Carlo Mariani (iD https://orcid.org/0000-0002-7979-1700

\section{References}

[1] Sofo J O, Chaudhari A S and Barber G D 2007 Phys. Rev. B 75 153401

[2] Samarakoon D K and Wang X Q 2010 ACS Nano 4 4126-30

[3] Shkrebtii A I, Heritage E, McNelles P, Cabellos J L and Mendoza B S 2012 Physica Status Solidi c 9 1378-83

[4] Gong K, Du F, Xia Z, Durstock M and Dai L 2009 Science 323 760-4

[5] Bacon M, Bradley S J and Nann T 2014 Part. Part. Syst. Charact. 31 415-28

[6] Hassoun J et al 2014 Nano Lett. 14 4901-6

[7] Massimi L et al 2015 J. Phys. Chem. C 119 2427-37

[8] Della Pia A et al 2016 J. Phys. Chem. C 120 7323-31

[9] Ong W J, Tan L L, Ng Y H, Yong S T and Chai S P 2016 Chem. Rev. 116 7159-329

[10] Ji Y, Dong H, Lin H, Zhang L, Hou T and Li Y 2016 RSC Adv. 6 52377-83

[11] Subrahmanyam K S, Kumar P, Maitra U, Govindaraj A, Hembram K P S S, Waghmare U V and Rao C N R 2011 Proc. Natl Acad. Sci. 108 2674-7

[12] Betti M G et al 2019 Prog. Part. Nucl. Phys. 106 120-31

[13] Betti M G et al 2019 J. Cosmol. Astropart. Phys. 2019047

[14] Sha X and Jackson B 2002 Surf. Sci. 496 318-30

[15] Hornekær L, Rauls E, Xu W, Śljivančanin I C V, Otero R, Stensgaard I, Lægsgaard E, Hammer B and Besenbacher F 2006 Phys. Rev. Lett. 97186102

[16] Boukhvalov D W, Katsnelson M I and Lichtenstein A I 2008 Phys. Rev. B 77035427

[17] Luo Z, Yu T, Kim K J, Ni Z, You Y, Lim S, Shen Z, Wang S and Lin J 2009 ACS Nano 3 1781-8

[18] Ruffieux P, Gröning O, Bielmann M, Mauron P, Schlapbach L and Gröning P 2002 Phys. Rev. B 66245416 
[19] Elias D C et al 2009 Science 323 610-3

[20] Ryu S, Han M Y, Maultzsch J, Heinz T F, Kim P, Steigerwald M L and Brus L E 2008 Nano Lett. 8 4597-602

[21] Felten A, McManus D, Rice C, Nittler L, Pireaux J J and Casiraghi C 2014 Appl. Phys. Lett. 105183104

[22] Burgess J S, Matis B R, Robinson J T, Bulat F A, Perkins F K, Houston B H and Baldwin J W 2011 Carbon 49 4420-6

[23] Whitener K E, Lee W K, Campbell P M, Robinson J T and Sheehan P E 2014 Carbon 72 348-53

[24] Son J, Lee S, Kim S J, Park B C, Lee H K, Kim S, Kim J H, Hong B H and Hong J 2016 Nat. Commun. 713261

[25] Luo Z et al 2010 Appl. Phys. Lett. 97233111

[26] Haberer D et al 2010 Nano Lett. $103360-6$

[27] Haberer D et al 2011 Physica Status Solidi b 248 2639-43

[28] Balog R, Andersen M, Jørgensen B, Sljivancanin Z, Hammer B, Baraldi A, Larciprete R, Hofmann P, Hornekær L and Lizzit S 2013 ACS Nano 7 3823-32

[29] Panahi M, Solati N and Kaya S 2019 Surf. Sci. 679 24-30

[30] Ito Y et al 2014 Angew. Chem. Int. Ed. 53 4822-6

[31] Di Bernardo I et al 2017 ACS Omega 2 3691-7

[32] Di Bernardo I et al 2018 Carbon 131 258-65

[33] Ito Y, Qiu H J, Fujita T, Tanabe Y, Tanigaki K and Chen M 2014 Adv. Mater. 26 4145-50

[34] Ito Y, Cong W, Fujita T, Tang Z and Chen M 2015 Angew. Chem. Int. Ed. 54 2131-6

[35] Ito Y, Tanabe Y, Han J, Fujita T, Tanigaki K and Chen M 2015 Adv. Mater. 27 4302-7

[36] Mazzucato S, Nardin D, Capizzi M, Polimeni A, Frova A, Seravalli L and Franchi S 2005 Mater. Sci. Eng. C 25 830-4 current trends in nanoscience-from Materials to Applications Proc. of the European Materials Research Society 2004-Symposium G

[37] Tedeschi D et al 2019 Adv. Mater. 311903795

[38] Blundo E, Di Giorgio C, Pettinari G, Yildirim T, Felici M, Lu Y, Bobba F and Polimeni A 2020 Adv. Mater. Interfaces 72000621

[39] Felton J et al 2020 Molecules 252526

[40] Massimi L, Angelucci M, Gargiani P, Betti M G, Montoro S and Mariani C 2014 J. Chem. Phys. 140244704

[41] Massimi L, Betti M G, Caramazza S, Postorino P, Mariani C, Latini A and Leardini F 2016 Nanotechnology 27435601

[42] Leardini F et al 2018 Nanotechnology 29025603

[43] Leardini F et al 2019 2D Mater. 6035015

[44] Blundo E, Felici M, Yildirim T, Pettinari G, Tedeschi D, Miriametro A, Liu B, Ma W, Lu Y and Polimeni A 2020 Phys. Rev. Res. 2012024
[45] Lacovig P, Pozzo M, Alfe D, Vilmercati P, Baraldi A and Lizzit S 2009 Phys. Rev. Lett. 103166101

[46] Scardamaglia M, Forte G, Lizzit S, Baraldi A, Lacovig P, Larciprete R, Mariani C and Betti M G 2011 J. Nanopart. Res. 13 6013-20

[47] Scardamaglia M, Lisi S, Lizzit S, Baraldi A, Larciprete R, Mariani C and Betti M G 2013 J. Phys. Chem. C 117 3019-27

[48] Eberlein T, Bangert U, Nair R R, Jones R, Gass M, Bleloch A L, Novoselov K S, Geim A and Briddon P R 2008 Phys. Rev. B 77233406

[49] Politano A, Campi D, Formoso V and Chiarello G 2013 Phys. Chem. Chem. Phys. 15 11356-61

[50] Di Filippo G, Liscio A and Ruocco A 2020 Appl. Surf. Sci. 512 145605

[51] Stankovich S, Dikin D A, Piner R D, Kohlhaas K A, Kleinhammes A, Jia Y, Wu Y, Nguyen S T and Ruoff R S 2007 Carbon 45 1558-65

[52] Shin Y E, Sa Y J, Park S, Lee J, Shin K H, Joo S H and Ko H 2014 Nanoscale 6 9734-41

[53] Jiménez-Arévalo N, Leardini F, Ferrer I J, Ares J R, Sánchez C, Saad Abdelnabi M M, Betti M G and Mariani C 2020 ACS Appl. Energy Mater. 3 1922-32

[54] Wojtaszek M, Tombros N, Caretta A, van Loosdrecht P H M and van Wees B J $2011 \mathrm{~J}$. Appl. Phys. 110063715

[55] Sun Z, Pint C L, Marcano D C, Zhang C, Yao J, Ruan G, Yan Z, Zhu Y, Hauge R H and Tour J M 2011 Nat. Commun. 2559

[56] Piazza F, Gough K, Monthioux M, Puech P, Gerber I, Wiens R, Paredes G and Ozoria C 2019 Carbon 145 10-22

[57] Jiang H, Kammler M, Ding F, Dorenkamp Y, Manby F R, Wodtke A M, Miller T F, Kandratsenka A and Bünermann O 2019 Science 364 379-82

[58] Barinov A, Gregoratti L, Dudin P, La Rosa S and Kiskinova M 2009 Adv. Mater. 21 1916-20

[59] Scardamaglia M, Amati M, Llorente B, Mudimela P, Colomer J F, Ghijsen J, Ewels C, Snyders R, Gregoratti L and Bittencourt C 2014 Carbon 77 319-28

[60] Susi T, Kaukonen M, Havu P, Ljungberg M P, Ayala P and Kauppinen E I 2014 Beilstein J. Nanotechnol. 5 121-32

[61] D'Acunto G, Ripanti F, Postorino P, Betti M G, Scardamaglia M, Bittencourt C and Mariani C 2018 Carbon $139768-75$

[62] Paris A et al 2013 Adv. Funct. Mater. 23 1628-35 